\title{
Coherent Beam Combining of a Colliding Pulse Modelocked VECSEL
}

\section{Conference Paper}

Author(s):

Waldburger, Dominik; Link, Sandro M.; Alfieri, Cesare G.E.; Golling, Matthias; Keller, Ursula (D)

Publication date:

2017

Permanent link:

https://doi.org/10.3929/ethz-b-000231075

Rights / license:

In Copyright - Non-Commercial Use Permitted

Originally published in:

https://doi.org/10.1364/ASSL.2017.AW4A.5 


\title{
Coherent Beam Combining of a Colliding Pulse Modelocked VECSEL
}

\author{
Dominik Waldburger, Sandro M. Link, Cesare G. E. Alfieri, Matthias Golling, and Ursula Keller \\ Department of Physics, Institute for Quantum Electronics, ETH Zürich, Auguste-Piccard-Hof 1, 8093 Zürich, Switzerland \\ dominikw@phys.ethz.ch
}

\begin{abstract}
We demonstrate coherent beam combining of a SESAM-modelocked VECSEL using additional passive stabilization of the two output beams from a ring cavity with colliding pulse modelocking.

OCIS codes: (140.5960) Semiconductor lasers; (140.7270) Vertical emitting lasers; (140.4050) Mode-locked lasers; (140.3480) Lasers, diode-pumped;
\end{abstract}

\section{Introduction}

Ultrafast optically pumped semiconductor disk lasers (SDLs) have shown an extraordinary progress over the last 10 years [1]. Pulse peak power of up to $6.3 \mathrm{~kW}$ [2] and pulse duration as short as $96 \mathrm{fs}$ [3] have been achieved. They convert low-coherent light from high-power diode pump arrays into diffraction-limited beams and cover the wavelength range from ultraviolet to the mid-infrared by combining bandgap engineering with the appropriate choice of the semiconductor material. Exciting application in the field of biomedical imaging and frequency comb metrology require the combination of short pulse duration and high pulse peak power to drive non-linear processes. To date ultrafast SDLs have shown a trade-off between pulse duration and average output power [4]. A particular application is the generation of a coherent octave-spanning supercontinuum which allows for self-referenced stabilization of the optical frequency comb [5], which is needed for most frequency comb applications. The required combination of 100fs pulses with kilowatts of pulse peak power, however, has only been achieved with external amplification and compression so far [6].

Here we demonstrate coherent beam combining from a SESAM-modelocked vertical external-cavity surfaceemitting laser (VECSEL) [7] in a ring cavity. Moloney and Stolz have recently demonstrated a colliding pulse modelocked (CPM) VECSEL in a ring cavity for the first time [8]. With pulses as short as $128 \mathrm{fs}$ and $90 \mathrm{~mW}$ average output power [9] the performance is comparable to the state-of-the-art results obtained in a linear V-shaped cavity [3]. Our goal here is to coherently combine the two output beams from the ring cavity to double the peak power for more efficient supercontinuum generation. However, coherent beam combining of the two output beams requires identical pulse repetition rates and carrier envelop offset (CEO) frequencies of the two output beams. Here we show that actually both the pulse repetition rates and the CEO frequencies of the two output beams are passively locked when the SESAM modelocked VECSEL is operated in the CPM [10].

\section{Measurement and Results}

In the CPM ring cavity (Fig. 1a), the laser gain is provided by a VECSEL chip which is passively modelocked with a semiconductor saturable absorber mirror (SESAM) [11]. A flat high reflectance mirror and an output coupler complete the cavity. The details of the used VECSEL and SESAM chip can be found in [3].The two counter-propagating pulses pass only once per round trip on the VECSEL gain chip and not twice as in the V-shaped cavity, which lowers the gain per round trip. For a CPM ring cavity the two counter-propagating pulses collide on the SESAM and their interference leads to a stronger loss saturation [9]. The distance between the VECSEL and the SESAM is set to one quarter of the total cavity length for equal time intervals between the pulses on the VECSEL chip. An intracavity Brewster plate is used to fix the polarization. The laser is operated in a configuration optimized for performance stability over weeks of operation rather than for short pulse generation. The pulse characteristics of the two output beams are identical with pulse durations of $780 \mathrm{fs}$ at repetition rates of $1.668 \mathrm{GHz}$ and center wavelengths of $1034 \mathrm{~nm}$. The average power per beam is $170 \mathrm{~mW}$.

First, we verify that the two pulse repetition rates are locked when CPM is working. To investigate the locking of the repetition rates, we stabilized the repetition rate of one beam to an electronic reference with a phase-locked loop that acts on the cavity length via an output coupler mounted on a piezo actuator. The feedback leads to a reduction of the measured in-loop phase noise by over $100 \mathrm{~dB}$ compared to free-running operation (Fig. 1b). At the same time, also the phase noise of the repetition rate of the other beam is reduce by the same amount, confirming that both pulse repetition rates are locked. 

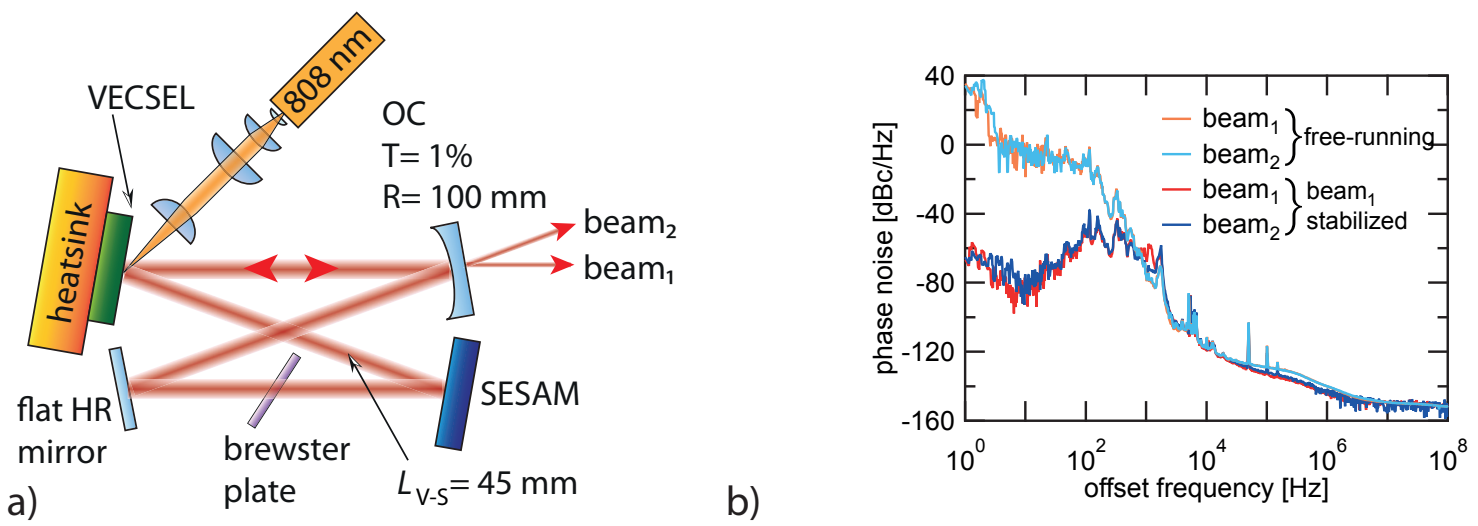

Fig. 1. a) Schematic of the colliding pulse modelocked (CPM) ring cavity comprising the VECSEL chip, the SESAM, a flat high reflectance (HR) mirror and an output coupler (OC). b) Phase noise measurement of the pulse repetition rate of the two output beams. If the pulse repetition rate of one beam is stabilized, the noise is drastically reduced simultaneously for both beams, confirming the locking of the pulse repetition rates.

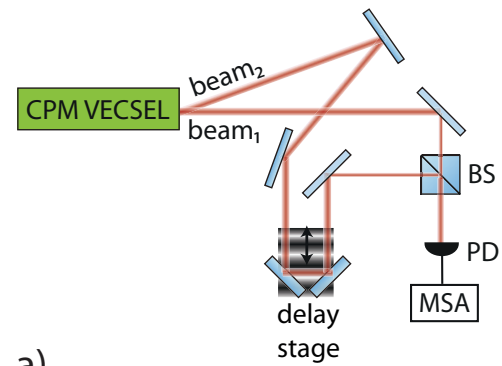

a)

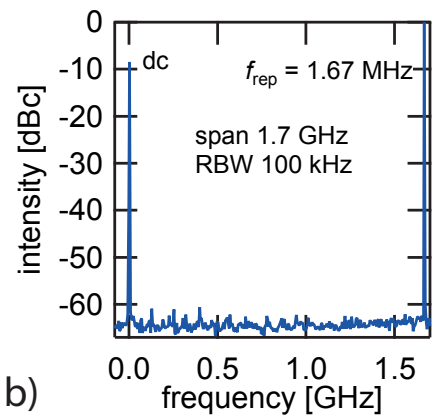

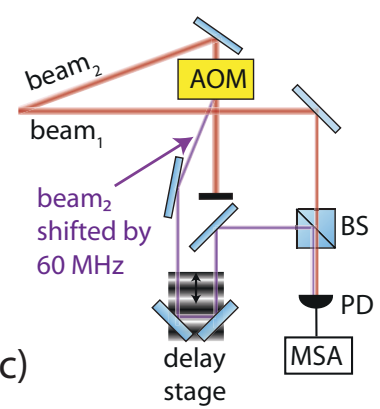

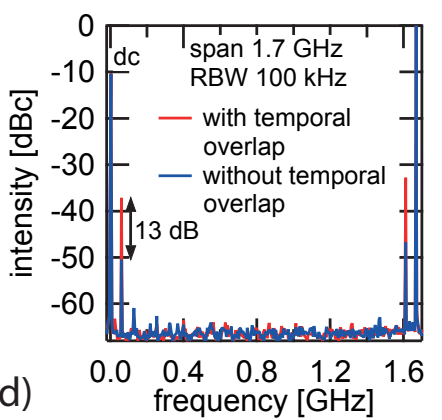

e)

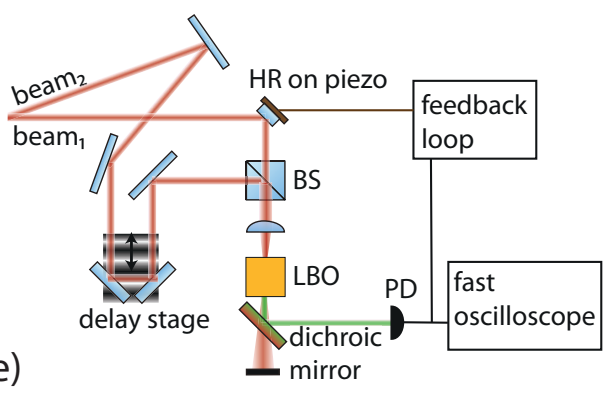

Fig. 2. a) Setup used to overlap the two beams temporally and spatially for the detection CEO frequency difference of the two beams. b) Measured microwave spectrum indicating that a zero difference in CEO frequencies due to the absence of a beat frequency. c) This is confirmed by shifting the frequency comb of one beam with an acousto-optic modulator (AOM). d) The measured beat signal at $60 \mathrm{MHz}$ (red line), which is stronger than the peak coming from the strong radio frequency driver of the AOM (blue line), confirms the locking of the CEO frequencies. e) Proof-of-principle SHG experiment of coherent pulse combining. f) Generated SHG signal for each beam individually (I \& II), for non-coherent combining (III), coherent combining (IV) and with active path stabilization (V). BS, beam splitter; PD, photodetector; MSA microwave spectrum analyzer; HR, high reflector; LBO, lithium triborate.

In a second step, we analyze the CEO frequency of both beams. For this, we use a delay stage and a 50:50 beam splitter to overlap the pulses of the two output beams in time and space (Fig. 2a). The interference of the two beams is recorded with a fast photodiode and analyzed with a microwave spectrum analyzer (MSA). Since the pulse repetition rates of the two beams are fixed, we expect to detected a beat signal corresponding to the difference in CEO frequencies of the two beams. Since we detect no beat signal (Fig. 2b), the difference in CEO frequencies is either varying so fast that it is below the noise-level of the MSA or the difference is exactly zero. To prove that the difference in CEO frequencies is actual zero and the CEO frequencies are locked, we use an acousto-optic modulator (AOM) with a modulation frequency of $60 \mathrm{MHz}$. As a result, the optical frequencies of the first order deflected beam of the 
AOM are shifted by $60 \mathrm{MHz}$ (Fig. 2c). In this case, we observe a beat signal at $60 \mathrm{MHz}$ on top of a signal originated from the strong radio frequency signal of the AOM driver (Fig. 2d). This measurement reveals that both CEO frequencies are perfectly synchronized.

Finally, we demonstrate the possibility of coherent pulse combining of the two beams in a proof-of-principle SHG experiment. We focus the temporal and spatial overlapping pulses in a non-critical phase-matched 20 -mm long lithium triborate (LBO) crystal for SHG (Fig. 3e). The used 50:50 beam splitter acts as a Mach-Zehnder-interferometer: For perfect overlap, we have total destructive interference in one arm and total constructive interference in the other arm. We achieve a maximal 7.7-fold improvement of the SHG signal compared to the non-coherent combined case, i.e. without temporal overlap, which is close to the theoretical maximum of 8 (Fig. 2f). The SHG signal is strongly oscillating as it is sensitive to path length differences of lambda half $(517 \mathrm{~nm})$. With an active path length stabilization, we could stabilize the SHG signal. Further applications will need a more mechanically stable setup and boxing to avoid air disturbances.

\section{Conclusion and Outlook}

We demonstrated that the CEO frequencies and pulse repetition rates of the two output beams of a CPM VECSEL are intrinsically locked. This enables coherent beam combining of the two output beams from the ring cavity, which we showed in a proof-of-principle experiment. In a future step, we will optimize the performance of the CPM laser for shorter pulses and higher peak power. The peak power of the coherent combination of such pulses should provide coherent octave-spanning supercontinuum generation in silicon nitride waveguides [12] for self-referenced optical frequency comb stabilization.

\section{Acknowledgement}

The authors acknowledge support of the technology and clean room facility FIRST of ETH Zurich for advanced microand nanotechnology. This work was financed by the Swiss Confederation Program Nano-Tera.ch, which was scientifically evaluated by the Swiss National Science Foundation (SNSF).

\section{References}

[1] Tilma, B. W., Mangold, M., Zaugg, C. A., Link, S. M., Waldburger, D., Klenner, A., Mayer, A. S., Gini, E., Golling, M., and Keller, U., "Recent advances in ultrafast semiconductor disk lasers," Light Sci. Appl. 4 (2015).

[2] Baker, C. W., Scheller, M., Laurain, A., Ruiz-Perez, A., Stolz, W., Addamane, S., Balakrishnan, G., Koch, S. W., Jones, R. J., and Moloney, J. V., "Multi-Angle VECSEL Cavities for Dispersion Control and Peak-Power Scaling," IEEE Photonics Technology Letters 29, 326-329 (2017).

[3] Waldburger, D., Link, S. M., Mangold, M., Alfieri, C. G. E., Gini, E., Golling, M., Tilma, B. W., and Keller, U., "High-power 100 fs semiconductor disk lasers," Optica 3, 844-852 (2016).

[4] Alfieri, C. G. E., Waldburger, D., Link, S. M., Gini, E., Golling, M., Eisenstein, G., and Keller, U., "Optical efficiency and gain dynamics of modelocked semiconductor disk lasers," Optics Express 25, 6402-6420 (2017).

[5] Telle, H. R., Steinmeyer, G., Dunlop, A. E., Stenger, J., Sutter, D. H., and Keller, U., "Carrier-envelope offset phase control: A novel concept for absolute optical frequency measurement and ultrashort pulse generation," Appl. Phys. B 69, 327-332 (1999).

[6] Zaugg, C. A., Klenner, A., Mangold, M., Mayer, A. S., Link, S. M., Emaury, F., Golling, M., Gini, E., Saraceno, C. J., Tilma, B. W., and Keller, U., "Gigahertz self-referenceable frequency comb from a semiconductor disk laser," Opt. Express 22, 16445-16455 (2014).

[7] Kuznetsov, M., Hakimi, F., Sprague, R., and Mooradian, A., "High-Power ( $>0.5-\mathrm{W}$ CW) Diode-Pumped Vertical-External-Cavity SurfaceEmitting Semiconductor Lasers with Circular TEM00 Beams," IEEE Photon. Technol. Lett. 9, 1063-1065 (1997).

[8] Laurain, A., Marah, D., Rockmore, R., McInerney, J., Hader, J., Perez, A. R., Stolz, W., and Moloney, J. V., "Colliding pulse mode locking of vertical-external-cavity surface-emitting laser," Optica 3, 781-784 (2016).

[9] Laurain, A., Rockmore, R., Chan, H.-T., Hader, J., Koch, S. W., Perez, A. R., Stolz, W., and Moloney, J. V., "Pulse interactions in a colliding pulse mode-locked vertical external cavity surface emitting laser," Journal of the Optical Society of America B 34, 329-337 (2017).

[10]Fork, R. L., Greene, B. I., and Shank, C. V., "Generation of optical pulses shorter than 0.1 ps by colliding pulse modelocking," Appl. Phys. Lett. 38, 617-619 (1981).

[11]Keller, U., Weingarten, K. J., Kärtner, F. X., Kopf, D., Braun, B., Jung, I. D., Fluck, R., Hönninger, C., Matuschek, N., and Aus der Au, J., "Semiconductor saturable absorber mirrors (SESAMs) for femtosecond to nanosecond pulse generation in solid-state lasers," IEEE J. Sel. Top. Quantum Electron. 2, 435-453 (1996).

[12]Klenner, A., Mayer, A. S., Johnson, A. R., Luke, K., Lamont, M. R. E., Okawachi, Y., Lipson, M., Gaeta, A. L., and Keller, U., "Gigahertz frequency comb offset stabilization based on supercontinuum generation in silicon nitride waveguides," Opt. Express 24, 11043-11053 (2016). 